\title{
LIGHT-TAILED ASYMPTOTICS OF STATIONARY PROBABILITY VECTORS OF MARKOV CHAINS OF GI/G/1 TYPE
}

\author{
QUAN-LIN LI,* Tsinghua University \\ YIQIANG Q. ZHAO,** Carleton University
}

\begin{abstract}
In this paper, we consider the asymptotic behavior of stationary probability vectors of Markov chains of GI/G/1 type. The generating function of the stationary probability vector is explicitly expressed by the $R$-measure. This expression of the generating function is more convenient for the asymptotic analysis than those in the literature. The $R G$-factorization of both the repeating row and the Wiener-Hopf equations for the boundary row are used to provide necessary spectral properties. The stationary probability vector of a Markov chain of GI/G/1 type is shown to be light tailed if the blocks of the repeating row and the blocks of the boundary row are light tailed. We derive two classes of explicit expression for the asymptotic behavior, the geometric tail, and the semigeometric tail, based on the repeating row, the boundary row, or the minimal positive solution of a crucial equation involved in the generating function, and discuss the singularity classes of the stationary probability vector.
\end{abstract}

Keywords: Markov chain of GI/G/1 type; Markov chain of GI/M/1 type; Markov chain of $\mathrm{M} / \mathrm{G} / 1$ type; asymptotic analysis; light tail; censoring method; $R G$-factorization; batch Markov arrival process

2000 Mathematics Subject Classification: Primary 60K25; 60K15

Secondary $60 \mathrm{~J} 22$

\section{Introduction}

Consider a Markov chain whose transition probability matrix is given by

$$
P=\left(\begin{array}{ccccc}
D_{0} & D_{1} & D_{2} & D_{3} & \cdots \\
D_{-1} & A_{0} & A_{1} & A_{2} & \cdots \\
D_{-2} & A_{-1} & A_{0} & A_{1} & \ldots \\
D_{-3} & A_{-2} & A_{-1} & A_{0} & \cdots \\
\vdots & \vdots & \vdots & \vdots & \ddots
\end{array}\right)
$$

where the matrices $A_{i},-\infty<i<\infty, D_{0}$, and $D_{j}$ and $D_{-j}, j \geq 1$, are of sizes $m \times m$, $m_{0} \times m_{0}, m_{0} \times m$, and $m \times m_{0}$, respectively. This Markov chain is referred to as being of GI/G/1 type. Throughout the paper, the Markov chain of GI/G/1 type is assumed to be irreducible and positive recurrent, and its stationary probability vector $\pi$ is partitioned accordingly into vectors $\left(\pi_{0}, \pi_{1}, \pi_{2}, \ldots\right)$.

Received 17 September 2002; revision received 15 August 2005.

* Postal address: Department of Industrial Engineering, Tsinghua University, Beijing, 100084, P. R. China.

** Postal address: School of Mathematics and Statistics, Carleton University, Ottawa, Ontario, Canada K1S 5B6.

Email address: zhao@math.carleton.ca 
Markov chains of GI/G/1 type have been extensively investigated by several researchers, among whom are Asmussen [6], Grassmann and Heyman [14], Højgaard and Møller [17], Asmussen and Møller [7], and Zhao et al. [31]-[33]. Readers may also refer to two overviews, and references therein: [15] and [30]. Two important examples of Markov chains of GI/G/1 type are Markov chains of GI/M/1 type and M/G/1 type, details about which may be found in [23], [25], and [19].

Our main focus in this paper is on the light-tailed asymptotic behavior of $\left\{\pi_{k}\right\}$. A sequence $\left\{c_{k}\right\}$ of nonnegative scalars is called light tailed if

$$
\sum_{k=1}^{\infty} c_{k} \exp \{\varepsilon k\}<\infty \quad \text { for some } \varepsilon>0 .
$$

In analogy, a light-tailed sequence of nonnegative matrices is defined as follows.

Definition 1. A sequence $\left\{C_{k}\right\}$ of nonnegative matrices of size $m \times n$ is called light tailed if, for all $i=1,2, \ldots, m$ and $j=1,2, \ldots, n$, the sequences $\left\{C_{k}(i, j)\right\}$ of nonnegative scalars are light-tailed, where $C_{k}(i, j)$ is the $(i, j)$ th entry of $C_{k}$.

The investigations of the light-tailed asymptotic behavior of stationary probability vectors of block-structured Markov chains have been inspired by [28] and [26]. Neuts [23] provided an excellent overview of the asymptotic behavior of Markov chains of GI/M/1 type. To establish useful relations between the light-tailed asymptotics and the parameters of queueing models, Neuts [24] discussed the caudal characteristic curves of some queues by means of matrix-geometric solutions, and Bean et al. [9] analyzed the caudal characteristics of quasibirth-death processes and Markov chains of GI/M/1 type. Fujimoto et al. [13] obtained an important result on the asymptotics of quasi-birth-death processes with both infinitely many levels and infinitely many phases. Bean and Nielsen [8] considered the decay rate of discrete phase-type distributions when there are a countably infinite number of phases.

However, in contrast to Markov chains of GI/M/1 type, it is more difficult to analyze the asymptotics of stationary probability vectors of Markov chains of M/G/1 type. This difficulty is due to two basic facts: (i) the non-matrix-geometric iterative solution of stationary probability vectors of Markov chains of M/G/1 type makes the analysis more difficult, and (ii) the stationary probability vectors of Markov chains of M/G/1 type can be either light tailed or heavy tailed. The light-tailed asymptotics of stationary probability vectors of Markov chains of M/G/1 type was studied in [11], [3], [22], [10], [18], and [27], for example. The study of heavy-tailed asymptotics of stationary probability vectors of Markov chains of M/G/1 type is limited so far to [7] and [20].

The main contributions of this paper are twofold. The first is to present a novel approach to evaluating the light-tailed asymptotics of stationary probability vectors of Markov chains of GI/G/1 type. The key idea in this approach is the $R G$-factorization of both the repeating blocks and the Wiener-Hopf equations for the boundary blocks of the transition probability matrix. As will be shown in this paper, and was seen in [20], the $R G$-factorization plays a role in analyzing stationary probability vectors (including queue lengths) that is of similar importance to that played by the Wiener-Hopf factorization in analyzing waiting times. Using the $R G$-factorization and the Wiener-Hopf equations, we first express $\left\{\pi_{k}\right\}$ in terms of the $R$-measure, and then express the $R$-measure in terms of the blocks in the transition probability matrix of GI/G/1 type. This expression, together with necessary spectral properties of the matrices involved and the important relations $\phi_{R}=\phi_{A+}$ and $\phi_{R_{0}}=\phi_{D}$ (see Theorem 1 and 
Lemma 3) between certain radii of convergence, can be used to show that $\left\{\pi_{k}\right\}$ is light tailed if $\min \left\{\phi_{A+}, \phi_{D}\right\}>1$.

The second contribution of the paper is to explicitly present the tail asymptotics of $\left\{\pi_{k}\right\}$. By means of the $R G$-factorization, we first establish existence conditions for the equation $\operatorname{det}\left(I-R^{*}(z)\right)=0$ to have the minimal solution $\eta$ in the range $z>1$. We then show that the light-tailed asymptotics of $\left\{\pi_{k}\right\}$ is explicitly determined by the relations between the three scalar parameters $\phi_{A+}, \phi_{D}$, and $\eta$. Though explicit expressions for the stationary tail asymptotics of Markov chains of GI/G/1 type can be given in some cases, it could be very challenging or impossible in other cases, for example for some Markov chains of GI/G/1 type with $1<\min \left\{\phi_{A+}, \phi_{D}\right\} \leq \eta$. In this situation, we define three classes of sequences of nonnegative matrices (including vectors). Elements of the first two classes exhibit light-tailed asymptotics while those of the third exhibit heavy-tailed asymptotics. The classification of $\left\{\pi_{k}\right\}$ is discussed in terms of the classification of the repeating row and the boundary row. This method of classification was first introduced by Abate et al. [4] and Abate and Whitt [1], [2] to analyze the tail asymptotics of waiting times.

Our results are an improvement over existing ones for the asymptotic analysis of stationary probability vectors of block-structured Markov chains.

(i) How the boundary behavior of a Markov chain of GI/G/1 type can influence the tail asymptotics of $\left\{\pi_{k}\right\}$ is analyzed in this paper in more detail and more generally. This phenomenon was illustrated by Falkenberg [11] using examples of M/G/1 type.

(ii) The method employed in this paper avoids the imposition of a condition usually assumed in other analyses; see, for example, Condition (A.2) of [22], Theorem 3.5 of [11], or Theorem 5 of [3]. We also demonstrate that the condition is not necessary.

(iii) The analysis in this paper is based on an expression for the generating function of $\left\{\pi_{k}\right\}$ in terms of the $R$-measure, which is a more convenient form than those used by other researchers. This allows us to analyze a more general type of Markov chain, i.e. GI/G/1 type, and provide sharper results than those in the current literature.

The remainder of the paper is organized as follows. In Section 2, the stationary probability vector $\left\{\pi_{k}\right\}$ is expressed in terms of the $R$-measure and a condition on light-tailed asymptotics is given. In Section 3, existence conditions on the minimal solution $\eta, \eta>1$, to the equation $\operatorname{det}\left(I-R^{*}(z)\right)=0$ are discussed. In Section 4 , asymptotic expressions for $\left\{\pi_{k}\right\}$ are derived for $1<\eta<\max \left\{\phi_{A+}, \phi_{D}\right\}$. In Section 5, conditions on the repeating row and on the boundary row under which $\left\{\pi_{k}\right\}$ can be classified into three singularity classes are studied. In this section, we also comment on some special cases for which explicit expressions for tail asymptotics can be derived when the boundary matrices determine the tail asymptotics. Finally, an example, involving a batch Markov arrival process (BMAP), and concluding remarks are provided in Section 6. Throughout the paper, matrix inequalities are evaluated entrywise.

\section{A condition on light-tailed asymptotics}

In this section, we provide a condition under which $\left\{\pi_{k}\right\}$ is light tailed. As we expect, the condition is in terms of the tails of both the repeating blocks and the boundary blocks.

Consider an arbitrary discrete-time Markov chain $\left\{X_{n}, n=1,2, \ldots\right\}$ whose state space $S$ is partitioned as $S=\bigcup_{i=0}^{\infty} L_{i}$, where $L_{i}=\left\{(i, j), j=1,2, \ldots, m_{i}\right\}$. In a state $(i, j), i$ is referred to as the level and $j$ the phase. We also write $L_{\leq i}=\bigcup_{k=0}^{i} L_{k}$ and $L_{\geq i}$ for the 
complement of $L_{\leq(i-1)}$. We partition the transition probability matrix of the Markov chain according to level as $P=\left(P_{i, j}\right)$, where $P_{i, j}$ is a matrix of size $m_{i} \times m_{j}$.

We now define the $R$ - and $G$-measures. For $0 \leq i<j, R_{i, j}$ is defined as a matrix of size $m_{i} \times m_{j}$ whose $(r, s)$ th entry is the expected number of visits of the Markov chain to state $(j, s)$ before it hits any state in $L_{\leq(j-1)}$, given that the process starts in state $(i, r)$. For $i>j \geq 0$, $G_{i, j}$ is defined as a matrix of size $m_{i} \times m_{j}$ whose $(r, s)$ th entry is the probability of hitting state $(j, s)$ when the process enters $L_{\leq}(i-1)$ for the first time, given that the process starts in state $(i, r)$. The sets of the matrices $\left\{R_{i, j}\right\}$ and $\left\{G_{i, j}\right\}$ are referred to as the $R$-measure and the $G$-measure, respectively.

For the transition probability matrix of $\mathrm{GI} / \mathrm{G} / 1$ type, $R_{i, j}$ and $G_{i, j}$ depend only on the difference between $i$ and $j$, except for $R_{0, j}, j=1,2, \ldots$, and $G_{i, 0}, i=1,2, \ldots$ Thus, we can define $R_{k}=R_{i, j}, k=j-i, j>i>0$, and $G_{k}=G_{i, j}, k=i-j, i>j>0$. References on the $R$ - and $G$-measures, which are closely related to this study, include [14], [16], [31], [32], [33], [30], and [20].

Remark 1. For a Markov chain of $\mathrm{GI} / \mathrm{G} / 1$ type, the $R$ - and $G$-measures can be given by an algebraic method. Let

$$
P=\begin{aligned}
& L_{\leq n} \\
& L_{\geq(n+1)}
\end{aligned}\left(\begin{array}{cc}
L_{\leq n} & L_{\geq(n+1)} \\
V & U \\
V & Q_{1}
\end{array}\right) \text { for } n \geq 1,
$$

and let $P^{[n]}=Q_{0}+U \widehat{Q}_{1} V$, where $\widehat{Q}_{1}=\sum_{k=0}^{\infty} Q_{1}^{k}$. We denote by $P_{i, j}^{[n]}$ the $(i, j)$ th block of $P^{[n]}$, partitioned according to level. It was shown in [14] that $P_{n, n}^{[n]}, P_{n-i, n}^{[n]}$, and $P_{n, n-j}^{[n]}$ are independent of $n \geq 1$. Let

$$
\Phi_{0}=P_{n, n}^{[n]}, \quad \Phi_{i}=P_{n-i, n}^{[n]}, \quad \text { and } \quad \Phi_{-j}=P_{n, n-j}^{[n]},
$$

for $n \geq 1$ and $1 \leq i, j \leq n-1$. Then

$$
R_{i}=\Phi_{i}\left(I-\Phi_{0}\right)^{-1}, \quad G_{i}=\left(I-\Phi_{0}\right)^{-1} \Phi_{-i}, \quad i \geq 1 .
$$

Note that $\Phi_{0}$ is a matrix of size $m \times m$ whose $(r, s)$ th entry is the probability of hitting state $(i, s)$ when the process enters $L_{\leq i}$ for the first time, given that the process starts in state $(i, r)$.

Define the generating functions of the sequences $\left\{A_{k}\right\},\left\{R_{k}\right\}$, and $\left\{G_{k}\right\}$ by

$$
A^{*}(z)=\sum_{k=-\infty}^{\infty} z^{k} A_{k}, \quad R^{*}(z)=\sum_{k=1}^{\infty} z^{k} R_{k}, \quad \text { and } \quad G^{*}(z)=\sum_{k=1}^{\infty} z^{-k} G_{k},
$$

respectively. Note that $A^{*}(z)$ is a Laurent series, which is not usually the case in the standard matrix-analytic treatment; see, for example, [23], [25], or [19]. A more detailed discussion is provided in Section 6.

The following $R G$-factorization will be used in our study; see [30] or [33] for details:

$$
I-A^{*}(z)=\left[I-R^{*}(z)\right]\left(I-\Phi_{0}\right)\left[I-G^{*}(z)\right] .
$$

By $\phi_{R}, \phi_{G}, \phi_{A+}$, and $\phi_{A-}$ we denote the radii of convergence of $R^{*}(z), G^{*}(z), \sum_{k=1}^{\infty} z^{k} A_{k}$, and $\sum_{k=1}^{\infty} z^{-k} A_{-k}$, respectively. The following theorem provides important relations between the radii of convergence. 
Theorem 1. The radii of convergence satisfy $\phi_{R}=\phi_{A+}$ and $\phi_{G}=\phi_{A_{-}}$.

Proof. Since $\sum_{k=1}^{\infty} A_{-k}$ and $\sum_{k=1}^{\infty} A_{k}$ are substochastic, we have $0 \leq \phi_{A_{-}} \leq 1 \leq \phi_{A+}$ $\leq \infty$. In addition, that $R=\sum_{k=1}^{\infty} R_{k}$ and $G=\sum_{k=1}^{\infty} G_{k}$ are finite (see Theorem 4 of [31]) shows that $0 \leq \phi_{G} \leq 1 \leq \phi_{R} \leq \infty$. Note that $R^{*}(z)$ is a power series, $G^{*}(z)$ is a Laurent series with only negative powers of $z, R^{*}(z)$ is analytic for $|z|<\phi_{R}$, and $G^{*}(z)$ is analytic for $|z|>\phi_{G}$. Thus, it is easy to see from (3) that $\phi_{R}=\phi_{A+}$ and $\phi_{G}=\phi_{A_{-}}$, using the facts that $0 \leq \phi_{A_{-}} \leq 1 \leq \phi_{A+} \leq \infty$ and $0 \leq \phi_{G} \leq 1 \leq \phi_{R} \leq \infty$.

Lemma 1. If $\phi_{A+}>1$ then $\left\{R_{k}\right\}$ is light tailed.

The proof is obvious from Theorem 1. The following spectral properties of the matrices $G^{*}(z)$ and $R^{*}(z)$, which were given in Corollary 30 of [33], are important for us.

Lemma 2. If the Markov chain of GI/G/1 type is irreducible and positive recurrent, then all the solutions, if any, to $\operatorname{det}\left(I-R^{*}(z)\right)=0$ lie in the region $|z|>1$, and all the solutions, if any, to $\operatorname{det}\left(I-G^{*}(z)\right)=0$ lie in the region $|z| \leq 1$.

For the boundary blocks, we have the Wiener-Hopf equations as shown in [30], one of which is given by

$$
R_{0, k}\left(I-\Phi_{0}\right)=D_{k}+\sum_{i=1}^{\infty} R_{0, i+k}\left(I-\Phi_{0}\right) G_{i}, \quad k \geq 1 .
$$

Define the generating functions of the sequences $\left\{D_{k}\right\}$ and $\left\{R_{0, k}\right\}$ by $D^{*}(z)=\sum_{k=1}^{\infty} z^{k} D_{k}$ and $R_{0}^{*}(z)=\sum_{k=1}^{\infty} z^{k} R_{0, k}$, respectively. It follows from (4) that

$$
R_{0}^{*}(z)\left(I-\Phi_{0}\right)=D^{*}(z)+\sum_{k=1}^{\infty} z^{k} \sum_{i=1}^{\infty} R_{0, i+k}\left(I-\Phi_{0}\right) G_{i} .
$$

The second term on the right-hand side in the above equation is nonnegative for $z>0$, since, from (2), $\left(I-\Phi_{0}\right) G_{i}$ can be interpreted as a transition probability block $\Phi_{-i}$ in a censored transition probability matrix $P^{[n]}$. Under the assumption that $P$ is irreducible, the inverse of $\left(I-\Phi_{0}\right)$ exists. Noting that $\left(I-\Phi_{0}\right)^{-1}=\sum_{k=0}^{\infty}\left(\Phi_{0}\right)^{k} \geq 0$, we have

$$
R_{0}^{*}(z) \geq D^{*}(z)\left(I-\Phi_{0}\right)^{-1} \text { for } z>0 .
$$

However,

$$
\begin{aligned}
\sum_{k=1}^{\infty} z^{k} \sum_{i=1}^{\infty} R_{0, i+k}\left(I-\Phi_{0}\right) G_{i} & =\sum_{i=1}^{\infty} z^{-i}\left(\sum_{k=1}^{\infty} z^{i+k} R_{0, i+k}\right)\left(I-\Phi_{0}\right) G_{i} \\
& \leq R_{0}^{*}(z)\left(I-\Phi_{0}\right) G^{*}(z) \text { for } z>0
\end{aligned}
$$

which leads to $R_{0}^{*}(z)\left(I-\Phi_{0}\right)\left[I-G^{*}(z)\right] \leq D^{*}(z)$. Under the assumption of the irreducibility and positive recurrence of $P$, by Lemma $2 I-G^{*}(z)$ is invertible for any $z>1$. Since $G^{*}(z)$ is nonincreasing for $z \in(1, \infty)$, we can choose a $\delta>0$ small enough that $G^{*}(z) \leq G^{*}(1+\delta)$ for $z>1+\delta$. Then $I-G^{*}(z) \geq I-G^{*}(1+\delta)$ for $z>1+\delta$. Since $R_{0}^{*}(z)\left(I-\Phi_{0}\right) \geq 0$, from (5), we obtain

$$
R_{0}^{*}(z)\left(I-\Phi_{0}\right)\left[I-G^{*}(1+\delta)\right] \leq R_{0}^{*}(z)\left(I-\Phi_{0}\right)\left[I-G^{*}(z)\right] \leq D^{*}(z) \text { for } z>1+\delta .
$$


Noting that $\left[I-G^{*}(1+\delta)\right]^{-1}=\sum_{k=0}^{\infty}\left[G^{*}(1+\delta)\right]^{k} \geq 0$, we find that

$$
R_{0}^{*}(z) \leq D^{*}(z)\left[I-G^{*}(1+\delta)\right]^{-1}\left(I-\Phi_{0}\right)^{-1} \text { for } z>1+\delta,
$$

which, together with (6), implies that

$$
D^{*}(z)\left(I-\Phi_{0}\right)^{-1} \leq R_{0}^{*}(z) \leq D^{*}(z)\left[I-G^{*}(1+\delta)\right]^{-1}\left(I-\Phi_{0}\right)^{-1} \text { for } z>1+\delta .
$$

The following lemma follows from (7).

Lemma 3. Let $\phi_{R_{0}}$ and $\phi_{D}$ be the radii of convergence of $R_{0}^{*}(z)$ and $D^{*}(z)$, respectively. Then $\phi_{R_{0}}=\phi_{D}$. Furthermore, $\left\{R_{0, k}\right\}$ is light tailed if and only if $\phi_{D}>1$.

According to Equation (28) of [14], the stationary probability vectors $\left\{\pi_{k}\right\}$ can be expressed in terms of the $R$-measure:

$$
\pi_{k}=\pi_{0} R_{0, k}+\sum_{i=1}^{k-1} \pi_{i} R_{k-i}, \quad k \geq 1 .
$$

The generating function $\Pi^{*}(z)$, defined by $\Pi^{*}(z)=\sum_{k=1}^{\infty} z^{k} \pi_{k}$, is then

$$
\Pi^{*}(z)=\pi_{0} R_{0}^{*}(z)\left[I-R^{*}(z)\right]^{-1},
$$

given that both sides are defined.

Remark 2. If $\operatorname{det}\left(I-R^{*}(z)\right)=0$ has a solution $z$ such that $|z|>1$, then, by the continuity of an implicit function, there exists a solution $z_{0}$ such that

$$
\eta=\left|z_{0}\right|=\min \left\{|z|: \operatorname{det}\left(I-R^{*}(z)\right)=0\right\}>1,
$$

since $\operatorname{det}\left(I-R^{*}(1)\right) \neq 0$, by Lemma 2 . Furthermore, in the next section we shall show that such a $z_{0}$ can be positive.

The following theorem provides a condition sufficient (and in fact also necessary, as shown in [20]) for $\left\{\pi_{k}\right\}$ to be light tailed.

Theorem 2. If the Markov chain of GI/G/1 type is irreducible and positive recurrent and both $\left\{D_{k}\right\}$ and $\left\{A_{k}\right\}$ are light tailed, then $\left\{\pi_{k}\right\}$ is light tailed.

Proof. We denote by $\phi_{\Pi}$ the radius of convergence of $\Pi^{*}(z)$. If the Markov chain of GI/G/1 type is irreducible and positive recurrent, then it follows from (8) that

$$
\phi_{\Pi}=\min \left\{\phi_{R_{0}}, \phi_{R}, \eta\right\}
$$

where

$$
\eta=\left|z_{0}\right|=\min \left\{|z|: 1<|z| \leq \phi_{A+}, \operatorname{det}\left(I-R^{*}(z)\right)=0\right\}
$$

if $\operatorname{det}\left(I-R^{*}(z)\right)=0$ has a solution, and $\eta=\infty$ otherwise.

If both $\left\{D_{k}\right\}$ and $\left\{A_{k}\right\}$ are light tailed, then $\min \left\{\phi_{A+}, \phi_{D}\right\}>1$. It follows from Theorem 1 that $\phi_{R_{0}}=\phi_{D}>1$ and $\phi_{R}=\phi_{A+}>1$. Since Lemma 2 implies that either $1<\eta<\infty$ or $\eta=\infty$, we obtain $\phi_{\Pi}=\min \left\{\phi_{R_{0}}, \phi_{R}, \eta\right\}>1$ and, hence, $\left\{\pi_{k}\right\}$ is light tailed. This completes the proof. 


\section{Conditions on the existence of the minimal solution}

As discussed in Section 2, the minimal positive solution to $\operatorname{det}(I-R(z))=0$ plays an important role in evaluating the tail asymptotic behavior of $\left\{\pi_{k}\right\}$. In this section, we discuss conditions on the existence of a minimal positive solution.

Theorem 3. Suppose that the Markov chain of GI/G/1 type is irreducible and positive recurrent, and that the matrix $A=\sum_{k=-\infty}^{\infty} A_{k}$ is irreducible. If the set

$$
\Omega=\left\{z: 1<|z|<\phi_{A+}, \operatorname{det}\left(I-R^{*}(z)\right)=0\right\}
$$

is not empty, then there must exist a solution $\eta>1$ to $\operatorname{det}\left(I-R^{*}(z)\right)=0$ such that $\eta \leq\left|z_{0}\right|$ for any $z_{0} \in \Omega$.

Proof. To prove this theorem, we construct a new Markov chain,

$$
P_{\text {new }}=\left(\begin{array}{ccccc}
\widetilde{D}_{0} & \widetilde{D}_{1} & \widetilde{D}_{2} & \widetilde{D}_{3} & \ldots \\
D_{-1} & A_{0} & A_{1} & A_{2} & \cdots \\
D_{-2} & A_{-1} & A_{0} & A_{1} & \ldots \\
D_{-3} & A_{-2} & A_{-1} & A_{0} & \cdots \\
\vdots & \vdots & \vdots & \vdots & \ddots
\end{array}\right) .
$$

Here the matrices $D_{-k}, k \geq 1$, and $A_{l},-\infty<l<\infty$, are the same as that of $P$ given in (1) and the matrices $\widetilde{D}_{k}, k \geq 0$, are given by

$$
\widetilde{D}_{0}=\frac{1}{m} \mathrm{e}^{-\tau} E, \quad \widetilde{D}_{k}=\frac{\tau^{k}}{m k !} \mathrm{e}^{-\tau} E, \quad k \geq 1,
$$

where $E$ is the $m \times m$ matrix of 1 s and $\tau$ is a positive scalar.

Since every entry of $\widetilde{D}_{k}, k \geq 0$, is positive and the Markov chain $P$ in (1) is irreducible, it is obvious that $P_{\text {new }}$ is irreducible. Let $\widetilde{D}^{*}(z)=\sum_{k=1}^{\infty} z^{k} \widetilde{D}_{k}$. It is easy to check that $\phi_{\widetilde{D}}=\infty$. Therefore, $\sum_{k=1}^{\infty} k \widetilde{D}_{k}$ is finite, which, since $\phi_{A+}>1$ and the Markov chain $P$ in (1) is positive recurrent, implies that $P_{\text {new }}$ is positive recurrent.

Let $\left\{\tilde{\pi}_{k}\right\}$ be the stationary probability vector of $P_{\text {new }}$, which can be explicitly expressed in terms of the $R$-measure of $P_{\text {new }}$. Let $\left\{\widetilde{R}_{0, k}\right\}$ and $\left\{\widetilde{R}_{k}\right\}$ comprise the $R$-measure of $P_{\text {new }}$. It is easy to see, from (10) and (1), that $\widetilde{R}_{k}=R_{k}$ for all $k \geq 1$. Let $\widetilde{\Pi}^{*}(z)$ and $\widetilde{R}_{0}^{*}(z)$ be the generating functions of $\left\{\widetilde{\pi}_{k}\right\}$ and $\left\{\widetilde{R}_{0, k}\right\}$, respectively. It follows from (8) that

$$
\widetilde{\Pi}^{*}(z)=\widetilde{\pi}_{0} \widetilde{R}_{0}^{*}(z)\left[I-R^{*}(z)\right]^{-1}=\frac{1}{\operatorname{det}\left(I-R^{*}(z)\right)} \widetilde{\pi}_{0} \widetilde{R}_{0}^{*}(z) \operatorname{adj}\left(I-R^{*}(z)\right),
$$

where $\operatorname{adj}\left(I-R^{*}(z)\right)$ denotes the adjoint of $I-R^{*}(z)$. It follows from Lemma 3 and Theorem 1 that $\phi_{\widetilde{R}_{0}}=\phi_{\widetilde{D}}=\infty>\phi_{A+}$ and $\phi_{R}=\phi_{A+}$. Let $\phi_{\widetilde{\pi}}$ be the radius of convergence of the vector generating function $\widetilde{\Pi}^{*}(z)=\sum_{k=1}^{\infty} z^{k} \widetilde{\pi}_{k}$. Then, it follows from a standard result in complex analysis (see Theorem 17.13 of [21, pp. 389-390]) that $z=\phi_{\tilde{\pi}}$ is a singular point of $\widetilde{\Pi}^{*}(z)$. Since $\Omega$ is not empty, there exists a solution $z_{1}$, with $1<\left|z_{1}\right|<\phi_{A+}, \operatorname{to} \operatorname{det}\left(I-R^{*}(z)\right)=0$. This shows that $z=z_{1}$ is a singular point of the complex vector function

$$
\frac{1}{\operatorname{det}\left(I-R^{*}(z)\right)} \widetilde{\pi}_{0} \widetilde{R}_{0}^{*}(z) \operatorname{adj}\left(I-R^{*}(z)\right) .
$$

Therefore, we have $1<\phi_{\tilde{\pi}} \leq\left|z_{1}\right|<\phi_{A+}$, according to (9). 
If it were the case that $\operatorname{det}\left(I-R^{*}\left(\phi_{\tilde{\pi}}\right)\right) \neq 0$, then

$$
\frac{1}{\operatorname{det}\left(I-R^{*}(z)\right)} \widetilde{\pi}_{0} \widetilde{R}_{0}^{*}(z) \operatorname{adj}\left(I-R^{*}(z)\right)
$$

would be analytic at $z=\phi_{\tilde{\pi}}$, since $1<\phi_{\tilde{\pi}}<\min \left\{\phi_{A+}, \phi_{\widetilde{D}}\right\}$. Hence, $\widetilde{\Pi}^{*}(z)$ also would be analytic at $z=\phi_{\tilde{\pi}}$. This contradicts $z=\phi_{\tilde{\pi}}$ being a singular point of $\widetilde{\Pi}^{*}(z)$ and, so, cannot be true. This completes the proof.

We now discuss the existence of a positive solution to $\operatorname{det}\left(I-R^{*}(z)\right)=0$, allowing for the fact that $\phi_{A+}$ itself can be such a solution.

Let $r(z)$ be the maximal eigenvalue of $R^{*}(z)$ for $z \in\left[1, \phi_{A+}\right)$. We can extend the domain of $r(z)$ to include $z=\phi_{A+}$ by defining $r\left(\phi_{A+}\right)=\lim _{z \nearrow \phi_{A+}} r(z)$. Clearly, $r(z) \geq 0$ for $1 \leq z<\phi_{A+}$, since $R^{*}(z) \geq 0$.

Theorem 4. Suppose that the Markov chain of GI/G/1 type is irreducible and positive recurrent. The equation $\operatorname{det}(I-R(z))=0$ has

(i) a positive solution $\eta$, satisfying $1<\eta<\phi_{A+}$, if $r\left(\phi_{A+}\right)>1$;

(ii) positive solution $\phi_{A+}$ if $r\left(\phi_{A+}\right)=1$ and $\phi_{A+}<\infty$; and

(iii) no positive solution if $r\left(\phi_{A+}\right)<1$.

Proof. Let $f(z)=1-r(z)$. It follows from Theorem 23 of [33] that $r(1)<1$ and, hence, $f(1)=1-r(1)>0$.

(i) If $r\left(\phi_{A+}\right)>1$ then there are two possible cases.

Case I: $r\left(\phi_{A+}\right)=\infty$. In this case, there always exists a $\xi, 1<\xi<\phi_{A+}$, such that $1<r(\xi)<\infty$, since $r(z)$ is continuous at $z$ for $1<z<\phi_{A+}$. Thus, we have $f(\xi)=1-$ $r(\xi)<0$. Since $f(z)$ is continuous on $[1, \xi], f(1)>0$, and $f(\xi)<0$, there must exist a point $\eta \in(1, \xi)$ such that $f(\eta)=0$.

Case II: $1<r\left(\phi_{A+}\right)<\infty$. In this case, an analysis similar to that in case I shows that there always exists a point $\eta \in\left(1, \phi_{A+}\right)$ satisfying $f(\eta)=0$.

(ii) If $r\left(\phi_{A+}\right)=1$ and $\phi_{A+}<\infty$, it is obvious that $z=\phi_{A+}$ is a solution to $f(z)=0$.

(iii) If $r\left(\phi_{A+}\right)<1$, since $f(1) \geq f(z) \geq f\left(\phi_{A+}\right)>0$ there exists no positive solution to $\operatorname{det}(I-R(z))=0$, for $z>1$.

Remark 3. The only case missing from Theorem 4 is that in which (iv) $r\left(\phi_{A+}\right)=1$ and $\phi_{A+}=$ $\infty$. In this case, it is possible that there does not exist any positive solution to $\operatorname{det}(I-R(z))=0$, for $z>1$, for example if the Markov chain of GI/G/1 type satisfies the condition that $r(z)$ is strictly increasing for $z \in(a, \infty)$, with $a>1$.

\section{The asymptotics based on the solution $\eta$}

In this section, we assume that $\Omega$ is not empty. Then, according to Theorem 3 , there exists a positive solution $\eta>1$ to $\operatorname{det}\left(I-R^{*}(z)\right)=0$ that is minimal in the sense that $\eta \leq|z|$ for any $z \in \Omega$. We further assume that $\eta<\phi_{D}$, which, together with the fact that $\eta \in \Omega$, implies $1<\eta<\min \left\{\phi_{A+}, \phi_{D}\right\}$. In this case, it is clear that the tail asymptotics of $\left\{\pi_{k}\right\}$ is determined by $\eta$ only. We will derive explicit asymptotic expressions for $\left\{\pi_{k}\right\}$ based on $\eta$. 


\section{1. $A$ is irreducible}

In this subsection, we assume that the matrix $A$ is irreducible. If $1<\eta<\min \left\{\phi_{A+}, \phi_{D}\right\}$, it follows from (8) that, for any $z$ with $1<|z|<\eta$,

$$
\Pi^{*}(z)=\frac{1}{\operatorname{det}\left(I-R^{*}(z)\right)} \pi_{0} R_{0}^{*}(z) \operatorname{adj}\left(I-R^{*}(z)\right) .
$$

The analysis of the singularity of $\Pi^{*}(z)$ depends on that of the three functions [det $(I-$ $\left.\left.R^{*}(z)\right)\right]^{-1}, R_{0}^{*}(z)$, and $\operatorname{adj}\left(I-R^{*}(z)\right)$. Since $R_{0}^{*}(z)$ and $\operatorname{adj}\left(I-R^{*}(z)\right)$ are analytic for $|z|<\min \left\{\phi_{A+}, \phi_{D}\right\}$, they are analytic at $z=\eta$. Since $z=\eta$ is a singular point of $\left[\operatorname{det}\left(I-R^{*}(z)\right)\right]^{-1}$, it is thus also a singular point of $\Pi^{*}(z)$. With the aid of several lemmas, we will show that $z=\eta$ is a pole of $\Pi^{*}(z)$ of order 1 , and is the only singular point.

It follows from (3) that

$$
\begin{aligned}
\left\{z: 0<z<\phi_{A+}, \operatorname{det}\left(I-A^{*}(z)\right)=0\right\}= & \left\{z: 0<z<\phi_{A+}, \operatorname{det}\left(I-R^{*}(z)\right)=0\right\} \\
& \cup\left\{z: 0<z<\phi_{A+}, \operatorname{det}\left(I-G^{*}(z)\right)=0\right\},
\end{aligned}
$$

since $I-\Phi_{0}$ is invertible. If the Markov chain of GI/G/1 type is irreducible and positive recurrent, it follows from (12) and Lemma 2 that

$$
\left\{z: 1<z<\phi_{A+}, \operatorname{det}\left(I-A^{*}(z)\right)=0\right\}=\left\{z: 1<z<\phi_{A+}, \operatorname{det}\left(I-R^{*}(z)\right)=0\right\}
$$

and

$$
\left\{z: 0<z \leq 1, \operatorname{det}\left(I-A^{*}(z)\right)=0\right\}=\left\{z: 0<z \leq 1, \operatorname{det}\left(I-G^{*}(z)\right)=0\right\} .
$$

For $z>0$, we denote by $\chi(z), r(z)$, and $g(z)$ the maximal eigenvalues of the matrices $A^{*}(z), R^{*}(z)$, and $G^{*}(z)$, respectively. Let $r_{i}(z), 2 \leq i \leq m$, denote all other eigenvalues of $R^{*}(z)$. We denote by $u(z)$ and $v(z)$ the left and right eigenvectors of $R^{*}(z)$ corresponding to the eigenvalue $r(z)$, respectively. In a similar fashion, $s(z)$ and $t(z)$ are defined for $A^{*}(z)$.

To characterize the function $\left[\operatorname{det}\left(I-R^{*}(z)\right)\right]^{-1}$, we introduce the following lemma, which relates the singularities of $\left[\operatorname{det}\left(I-R^{*}(z)\right)\right]^{-1}$ to the roots of $\operatorname{det}\left(I-A^{*}(z)\right)=0$.

Lemma 4. Suppose that the Markov chain of GI/G/1 type is irreducible and positive recurrent. Then,

(i) $\min \left\{z: 1<z<\phi_{A+}, \operatorname{det}\left(I-R^{*}(z)\right)=0\right\}=\min \left\{z: 1<z<\phi_{A+}, r(z)=1\right\}$;

(ii) $\min \left\{z: 1<z<\phi_{A+}, \operatorname{det}\left(I-A^{*}(z)\right)=0\right\}=\min \left\{z: 1<z<\phi_{A+}, \chi(z)=1\right\}$;

(iii) $\min \left\{z: 1<z<\phi_{A+}, r(z)=1\right\}=\min \left\{z: 1<z<\phi_{A+}, \chi(z)=1\right\}$.

Proof. (i) Let $\Omega_{0}=\left\{z: 1<z<\phi_{A+}, r(z)=1\right\}$. It is clear that $\Omega_{0} \subset \Omega$, since $\operatorname{det}\left(I-R^{*}(z)\right)=[1-r(z)] \prod_{i=2}^{m}\left[1-r_{i}(z)\right]$ for $z>1$.

There are two possible cases for the set $\Omega$.

Case I: $\Omega$ is not empty. In this case, it follows from Theorem 3 that there exists an $\eta$ such that

$$
\eta=\min \left\{z: 1<z<\phi_{A+}, \operatorname{det}\left(I-R^{*}(z)\right)=0\right\}=\min \Omega .
$$

Suppose that $\eta \notin \Omega_{0}$, i.e. $r(\eta) \neq 1$. Since $\operatorname{det}\left(I-R^{*}(\eta)\right)=0$, there must exist an $r_{i_{0}}(\eta), 2 \leq$ $i_{0} \leq m$, such that $r_{i_{0}}(\eta)=1$. Noting that $r(\eta)$ is the maximal eigenvalue of $R^{*}(\eta)$, we therefore have $r(\eta) \geq r_{i_{0}}(\eta)=1$. By the assumption that $r(\eta) \neq 1$, we should have $r(\eta)>r_{i_{0}}(\eta)=1$. 
Let $f(z)=1-r(z)$. Then $f(z)$ is continuous for $z \in[1, \eta], f(1)>0$, and $f(\eta)<0$; hence, there exists a point $\xi \in(1, \eta)$ such that $f(\xi)=0$, i.e. $r(\xi)=1$. Clearly, $\xi \in \Omega$. This contradicts $\eta$ being the minimum in $\Omega$. Therefore, $r(\eta)=1$ or $\eta \in \Omega_{0}$.

Since $\Omega_{0} \subset \Omega$, we have $\min \Omega \leq \min \Omega_{0}$. Clearly, $\eta=\min \Omega_{0}$ follows from $\eta=\min \Omega$ and $\eta \in \Omega_{0}$. Therefore, $\eta=\min \Omega=\min \Omega_{0}$ implies that (14) is true.

Case II: $\Omega$ is empty. In this case, $\Omega_{0}$ also is empty, since $\Omega_{0} \subset \Omega$. Hence, (14) is true in the sense that both sides are infinite.

(ii) The proof is slightly different from that in (i). In this case, let $f(z)=1-\chi(z)$. Since $\chi(1)=1$, in order to make the argument used in (i) we must find a $z_{0}>1$ such that $\chi\left(z_{0}\right)<1$.

Since the Markov chain of GI/G/1 type is assumed to be both irreducible and positive recurrent and $\phi_{A+}>1$, it follows from Proposition 3.1 of [6, p. 318] that

$$
\omega \sum_{k=1}^{\infty} k\left(A_{k}-A_{-k}\right) e<0,
$$

where $\omega$ is the stationary probability vector of the matrix $A$ and $e$ is a column vector of ones. Let $u(z)$ be the left Perron-Frobenius eigenvector of $A^{*}(z)$. By differentiating $s(z) A^{*}(z)=$ $\chi(z) s(z)$, we immediately obtain

$$
\chi^{\prime}(1)=\omega \sum_{k=1}^{\infty} k\left(A_{k}-A_{-k}\right) e<0 .
$$

Hence, there exists a $\delta>0$ small enough that $\chi(1+\delta)<\chi(1)=1$. Let $z_{0}=1+\delta$; then $f\left(z_{0}\right)>0$. The rest of the proof is similar to that in (i).

(iii) Noting that, from (13),

$\min \left\{z: 1<z<\phi_{A+}, \operatorname{det}\left(I-R^{*}(z)\right)=0\right\}=\min \left\{z: 1<z<\phi_{A+}, \operatorname{det}\left(I-A^{*}(z)\right)=0\right\}$,

the proof follows from (i) and (ii).

This completes the proof.

The following lemma characterizes $u(\eta)$ and $v(\eta)$ using the left and right Perron-Frobenius eigenvectors of $A^{*}(\eta)$. The proof is obvious and is omitted here.

Lemma 5. If $s(\eta)$ and $t(\eta)$ are the left and right Perron-Frobenius eigenvectors of $A^{*}(\eta)$, respectively, then

(i) $u(\eta)=a s(\eta)$ and $v(\eta)=b\left(I-\Phi_{0}\right)\left[I-G^{*}(\eta)\right] t(\eta)$, where $a$ and $b$ are two arbitrary positive factors;

(ii) $u(\eta)>0$ if and only if $s(\eta)>0 ; v(\eta) \geq 0$ but $v(\eta) \neq 0$ if and only if $t(\eta)>0$; and $u(z) e=u(z) v(z)=1$.

To describe $\Pi^{*}(z)$ in (11) in detail, we must express the adjoint matrix of $I-R^{*}(\eta)$ more explicitly.

Lemma 6. If the Markov chain of GI/G/1 type is irreducible and positive recurrent, then

$$
\operatorname{adj}\left(I-R^{*}(\eta)\right)=\prod_{i=2}^{m}\left[1-r_{i}(\eta)\right] v(\eta) u(\eta) .
$$


Proof. Note that the adjoint matrix of any singular matrix has rank one whenever 0 has algebraic multiplicity one, which holds for $I-R^{*}(\eta)$. Some simple computations lead to the desired result.

Using the above lemmas, we can easily show the following corollary.

Corollary 1. The only singular point of $\Pi^{*}(z)$ on $|z|=\eta$ is $z=\eta$, and it is a pole of order 1 .

Proof. Since $A^{*}(\eta)$ is irreducible, $1-\chi(\eta)=0$ is a simple eigenvalue of $I-A^{*}(\eta)$ and, for any other eigenvalue $1-y(\eta)$ of $I-A^{*}(\eta)$, we have $|1-y(\eta)|>|1-\chi(\eta)|=0$. Therefore, on $|z|=\eta, z=\eta$ is the only root of $\operatorname{det}\left(I-R^{*}(\eta)\right)=0$.

Let

$$
1-r(z)=(\eta-z) h(z) .
$$

To prove that $z=\eta$ is a pole of order 1 , it is sufficient to show that $h(\eta)>0$. This can be done by noting that $h(\eta)=r^{\prime}(\eta)$, which follows from elementary calculations.

Remark 4. At this point, we have proved the Perron-Frobenius property for $R^{*}(\eta)$ : the eigenvalue of $R^{*}(\eta)$ with maximal real part is real and unique with corresponding left and right eigenvectors $u(\eta)>0$ and $v(\eta) \geq 0$, but $v(\eta) \neq 0$.

We now provide an expression for $\Pi^{*}(z)$ by means of Lemmas 5 and 6 . According to Lemma 6, we can write

$$
\operatorname{adj}\left(I-R^{*}(z)\right)=\prod_{i=2}^{m}\left[1-r_{i}(\eta)\right] v(\eta) u(\eta)+(\eta-z) H(z),
$$

where $H(z)$ is an analytic function for $z, 1<|z|<\eta$. It follows from (11) and (15) that

$$
\Pi^{*}(z)=\frac{1}{\eta-z} \frac{1}{h(z) \prod_{i=2}^{m}\left[1-r_{i}(z)\right]} \pi_{0} R_{0}^{*}(z)\left[\prod_{i=2}^{m}\left[1-r_{i}(\eta)\right] v(\eta) u(\eta)+(\eta-z) H(z)\right] .
$$

Let

$$
L(z)=\frac{\pi_{0} R_{0}^{*}(z) v(\eta) \prod_{i=2}^{m}\left[1-r_{i}(\eta)\right]}{h(z) \prod_{i=2}^{m}\left[1-r_{i}(z)\right]} .
$$

Since $h(z)$ is continuous at $z=\eta$ and $h(\eta)>0$, there exists a $\sigma>0$ small enough that $h(z)>0$ for all $z \in(\eta-\sigma, \eta+\sigma)$. Noting that $h(z), \prod_{i=2}^{m}\left[1-r_{i}(z)\right]$, and $R_{0}^{*}(z)$ are all analytic at $z=\eta$, it is obvious that $L(z)$ is analytic at $z=\eta$. Thus, a power expansion of $L(z)$ at $z=\eta$ is given by

$$
L(z)=\frac{\pi_{0} R_{0}^{*}(\eta) v(\eta)}{h(\eta)}+\sum_{k=1}^{\infty} \frac{L^{(k)}(\eta)}{k !}(\eta-z)^{k},
$$

where $L^{(k)}(\eta)=\left.\left(\mathrm{d}^{k} / \mathrm{d} z^{k}\right) L(z)\right|_{z=\eta}$. It follows from (16) and (17) that

$$
\Pi^{*}(z)=\frac{K}{\eta-z} u(\eta)+g(z)
$$

where

$$
K=\frac{\pi_{0} R_{0}^{*}(\eta) v(\eta)}{h(\eta)}
$$


is a constant and

$$
g(z)=\frac{\pi_{0} R_{0}^{*}(z)}{h(z) \prod_{i=2}^{m}\left[1-r_{i}(z)\right]} H(z)+\sum_{k=1}^{\infty} \frac{L^{(k)}(\eta)}{k !}(\eta-z)^{k-1} u(\eta) .
$$

This vector is analytic at $z=\eta$, since

$$
\frac{\pi_{0} R_{0}^{*}(z)}{h(z) \prod_{i=2}^{m}\left[1-r_{i}(z)\right]}, \quad H(z), \quad \text { and } \quad \sum_{k=1}^{\infty} \frac{L^{(k)}(\eta)}{k !}(\eta-z)^{k}
$$

are all analytic at $z=\eta$.

The following lemma introduces a useful property of the $R$-measure. The proof is based on standard probabilistic arguments and the irreducibility of $P$; see, for example, the proof of Lemma 1.2.4 of [23].

Lemma 7. If the Markov chain is irreducible then each column vector of the matrix $R_{0}^{*}(1)$ is nonzero.

Theorem 5. Suppose that the Markov chain of GI/G/1 type is irreducible and positive recurrent. If $1<\eta<\min \left\{\phi_{A+}, \phi_{D}\right\}$ and the matrix $A$ is irreducible, then the tail asymptotics of $\left\{\pi_{k}\right\}$ is geometric, i.e.

$$
\pi_{k}=K \eta^{-(k+1)} u(\eta)+O\left((\eta+\varepsilon)^{-k}\right) e,
$$

where $\varepsilon$ is a small positive number.

Proof. Using (18), we first need to check that $K>0$. Then (19) is obviously true, according to a standard result on the asymptotics of complex functions (see, for example, Theorem 5.2.1 of [29]).

According to the assumptions made on the Markov chain, every $\pi_{k}$ is positive. It is clear that $\pi_{0} R_{0}^{*}(\eta) \geq \pi_{0} R_{0}^{*}(1)>0$, because $\pi_{0}>0$ and, according to Lemma 7, no column vector of $R_{0}^{*}(1)$ is identically zero. Noting that $v(\eta) \geq 0$ and $v(\eta) \neq 0$, we obtain $\pi_{0} R_{0}^{*}(\eta) v(\eta) \geq$ $\pi_{0} R_{0}^{*}(1) v(\eta)>0$. That $K>0$ now follows from $h(\eta)>0$. This completes the proof.

\section{2. $A$ is reducible}

In this subsection, we assume that the matrix $A$ is reducible. In this case, it is possible that the tail asymptotics of $\left\{\pi_{k}\right\}$ is not geometric when $1<\eta<\min \left\{\phi_{A+}, \phi_{D}\right\}$.

After reordering the states, we assume that $A^{*}(z)$ is written in the normal form

$$
A^{*}(z)=\left(\begin{array}{ccccccc}
a_{1}(z) & & & & & & \\
& \ddots & & & & \\
& & a_{p}(z) & & & & \\
& & & a_{p+1}(z) & & & \\
& & & & \ddots & & \\
& & & & & a_{p+q}(z) & \\
c_{1}(z) & \cdots & c_{p}(z) & c_{p+1}(z) & \cdots & c_{p+q}(z) & c_{p+q+1}(z)
\end{array}\right),
$$

where $p+q \geq 1$, the $a_{i}(z), 1 \leq i \leq p+q$, are irreducible and stochastic, and the missing entries are all 0 . Assume that $\eta>1$ is a solution to $\operatorname{det}\left(I-a_{i}(z)\right)=0,1 \leq i \leq p$, and 
that $I-c_{p+q+1}(\eta)$ and $I-a_{p+j}(\eta), 1 \leq j \leq q$, are all invertible. It follows from (3) and Lemma 2 that, for $1<z<\eta$,

$$
I-R^{*}(z)=\left[I-A^{*}(z)\right]\left[I-G^{*}(z)\right]^{-1}\left(I-\Phi_{0}\right)^{-1} .
$$

Thus, we obtain

$$
\begin{aligned}
\operatorname{det}\left(I-R^{*}(z)\right)= & (\eta-z)^{p} \prod_{i=1}^{p} b_{i}(z) \prod_{j=1}^{q} \operatorname{det}\left(I-a_{p+j}(z)\right) \operatorname{det}\left(I-c_{p+q+1}(z)\right) \\
& \times\left[\operatorname{det}\left(I-G^{*}(z)\right)\right]^{-1}\left[\operatorname{det}\left(I-\Phi_{0}\right)\right]^{-1}
\end{aligned}
$$

where $\operatorname{det}\left(I-a_{i}(z)\right)=(\eta-z) b_{i}(z), 1 \leq i \leq p$. It follows from Lemma 5 that $b_{i}(\eta) \neq$ $0,1 \leq i \leq p$. Thus, it follows from (11) and (15) that

$$
\Pi^{*}(z)=\frac{1}{(\eta-z)^{p}} K(z)
$$

where

$$
\begin{aligned}
K(z)= & \left\{\prod_{i=1}^{p} b_{i}(z) \prod_{j=1}^{q} \operatorname{det}\left(I-a_{p+j}(z)\right) \operatorname{det}\left(I-c_{p+q+1}(z)\right)\right\}^{-1} \\
& \times\left[\operatorname{det}\left(I-G^{*}(z)\right)\right]\left[\operatorname{det}\left(I-\Phi_{0}\right)\right] \pi_{0} R_{0}^{*}(z) \operatorname{adj}\left(I-R^{*}(z)\right)
\end{aligned}
$$

is analytic at $z=\eta$. Let

$$
K(z)=\sum_{k=0}^{\infty} \frac{K^{(k)}(\eta)}{k !}(\eta-z)^{k}, \quad \text { where } K^{(k)}(\eta)=\left.\frac{\mathrm{d}^{k}}{\mathrm{~d} z^{k}} K(z)\right|_{z=\eta}
$$

Then

$$
\Pi^{*}(z)=\sum_{i=1}^{p} \frac{K^{(p-i)}(\eta)}{(\eta-z)^{i}}+\sum_{j=0}^{\infty} K^{(p+j)}(\eta)(\eta-z)^{j} .
$$

By a standard result on the asymptotics of complex functions (see, for example, Theorem 5.2.1 of [29]), we obtain

$$
\pi_{k}=\sum_{l=1}^{p}(-1)^{l} \eta^{-(k+l)}\left(\begin{array}{c}
k+l-1 \\
l-1
\end{array}\right) K^{(p-l)}(\eta)+O\left((\eta+\varepsilon)^{-k}\right) e .
$$

Remark 5. If $1<\eta<\min \left\{\phi_{A+}, \phi_{D}\right\}$ then (20) provides a more general result on the light tail of $\left\{\pi_{k}\right\}$. Let $\operatorname{det}\left(I-A^{*}(z)\right)=(\eta-z)^{p} T(z)$, where $T(\eta) \neq 0$. Then the tail of $\left\{\pi_{k}\right\}$ is geometric if $p=1$ and nongeometric if $p \geq 2$.

\section{Three classes of $\left\{\pi_{k}\right\}$}

In this section, we discuss Markov chains of GI/G/1 type under the condition that $\min \left\{\phi_{A+}, \phi_{D}\right\}>1$, or $\left\{\pi_{k}\right\}$ is light tailed. Frequently, it is very difficult or impossible to find an explicit asymptotic expression for the tail. In this case, we classify $\left\{\pi_{k}\right\}$ into three classes according to the characteristics of its singularities. We are able to use the classes of 
singularity of $\left\{D_{k}\right\}$ and/or $\left\{A_{k}\right\}$ to characterize these classes. This method was first used by Abate et al. [4] and Abate and Whitt [1], [2] to discuss the tail of the waiting time.

First, we define a collection of matrix sequences consisting of all nonnegative matrix sequences (not necessarily of square matrices) whose sum is convergent. Let

$$
\mathcal{R}=\left\{\left\{B_{k}\right\}: B_{k} \geq 0, \sum_{k=0}^{\infty} B_{k}<\infty\right\} .
$$

We classify the matrices in $\mathcal{R}$ into three classes that are closely related to the standard notions of $\alpha$-recurrence and $\alpha$-transience; for example, see [5].

Definition 2. For $\left\{B_{k}\right\} \in \mathcal{R}$, let $\phi_{B}$ be the radius of convergence of $B^{*}(z)=\sum_{k=0}^{\infty} z^{k} B_{k}$. Then,

(i) $\left\{B_{k}\right\}$ is said to be of class I if $\phi_{B}>1$ and $B^{*}\left(\phi_{B}\right)$ is infinite,

(ii) $\left\{B_{k}\right\}$ is said to be of class II if $\phi_{B}>1$ and $B^{*}\left(\phi_{B}\right)$ is finite, and

(iii) $\left\{B_{k}\right\}$ is said to be of class III if $\phi_{B}=1$.

It is obvious that if $\left\{B_{k}\right\}$ is class I or II then it is light tailed, while if it is class III then it is heavy tailed. Therefore, both $\left\{D_{k}\right\}$ and $\left\{A_{k}\right\}$ are either class I or class II if $\min \left\{\phi_{A+}, \phi_{D}\right\}>1$.

Recall that $\eta=\infty$ if $\operatorname{det}\left(I-R^{*}(z)\right) \neq 0$ for all $|z| \leq \phi_{\mathrm{A}}$. For the matrix sequence $\left\{R_{k}\right\}$, we define its convolution as $R_{k}^{2 *}=\sum_{j=1}^{k} R_{j} R_{k-j}$. We further define

$$
R_{k}^{n *}=\sum_{j=1}^{k} R_{j}^{*(n-1)} R_{k-j}
$$

for $n \geq 3$, with $R_{k}^{1 *}=R_{k}$ and $R_{k}^{0 *}=I$.

Lemma 8. Suppose that the Markov chain of GI/G/1 type is irreducible and positive recurrent.

(i) If $1<\eta<\infty$ and $\eta \leq \phi_{A+}$ then $\left\{\sum_{n=0}^{\infty} R_{k}^{n *}\right\}$ is class I.

(ii) If $\eta=\infty$ then $\left\{\sum_{n=0}^{\infty} R_{k}^{n *}\right\}$ is class I if $\left\{A_{k}\right\}$ is class I and class II if $\left\{A_{k}\right\}$ is class II.

(iii) If $\phi_{D}>1$ then $\left\{R_{0, k}\right\}$ is class I if $\left\{D_{k}\right\}$ is class I and class II if $\left\{D_{k}\right\}$ is class II.

Proof. We will prove parts (i) and (ii); part (iii) can be proved similarly.

To prove part (i), assume that $1<\eta<\infty$ and $\eta \leq \phi_{A+}$. In this case, the radius of convergence of

$$
\sum_{k=1}^{\infty} z^{k} \sum_{n=0}^{\infty} R_{k}^{n *}=\frac{1}{\operatorname{det}\left(I-R^{*}(z)\right)} \operatorname{adj}\left(I-R^{*}(z)\right)
$$

is $\eta$. When $z=\eta \operatorname{det}\left(I-R^{*}(\eta)\right)=0$ and $\operatorname{adj}\left(I-R^{*}(\eta)\right) \neq 0$ according to Lemma 6 . Thus, $\left\{\sum_{n=0}^{\infty} R_{k}^{n *}\right\}$ is class I. 
We now prove part (ii). Since $\phi_{R}=\phi_{A+}$ from Theorem 1, it follows from (3) that

$$
\begin{aligned}
\sum_{k=1}^{\infty} z^{k} \sum_{n=0}^{\infty} R_{k}^{n *} & =\left[I-R^{*}(z)\right]^{-1}=\left(I-\Phi_{0}\right)\left[I-G^{*}(z)\right]\left[I-A^{*}(z)\right]^{-1} \\
& =\left(I-\Phi_{0}\right)\left[I-G^{*}(z)\right] \sum_{n=0}^{\infty}\left[A^{*}(z)\right]^{n} .
\end{aligned}
$$

Thus, the radius of convergence of $\sum_{k=1}^{\infty} z^{k} \sum_{n=0}^{\infty} R_{k}^{n *}$ is $\phi_{A+}$. Clearly, if $\left\{A_{k}\right\}$ is class I then $A^{*}\left(\phi_{A+}\right)$ is infinite and, hence, $\sum_{n=0}^{\infty}\left[A^{*}\left(\phi_{A+}\right)\right]^{n}$ is infinite. Since $I-\Phi_{0}$ and $I-G^{*}\left(\phi_{A+}\right)$ are invertible, $\sum_{k=1}^{\infty} \phi_{A+}^{k} \sum_{n=0}^{\infty} R_{k}^{n *}$ is infinite. Therefore, $\left\{\sum_{n=0}^{\infty} R_{k}^{n *}\right\}$ is class I.

If $\left\{A_{k}\right\}$ is class II then $\phi_{A+}<\infty$ and $A^{*}\left(\phi_{A+}\right)<\infty$. Since $\eta=\infty$, it follows from (3) that $I-A^{*}\left(\phi_{A+}\right)$ is invertible. Thus,

$$
\sum_{k=1}^{\infty} \phi_{A+}^{k} \sum_{n=0}^{\infty} R_{k}^{n *}=\left(I-\Phi_{0}\right)\left[I-G^{*}\left(\phi_{A+}\right)\right]\left[I-A^{*}\left(\phi_{A+}\right)\right]^{-1}<\infty .
$$

Therefore, $\left\{\sum_{n=0}^{\infty} R_{k}^{n *}\right\}$ is class II.

Theorem 6. Suppose that the Markov chain of GI/G/1 type is irreducible and positive recurrent.

(i) If $1<\eta<\infty$ and $\eta \leq \min \left\{\phi_{A+}, \phi_{D}\right\}$ then $\left\{\pi_{k}\right\}$ is class $I$.

(ii) If $\eta=\infty$ and $1<\phi_{A+}<\phi_{D}$, then $\left\{\pi_{k}\right\}$ is class I if $\left\{A_{k}\right\}$ is class I and class II if $\left\{A_{k}\right\}$ is class II.

(iii) If $\eta=\infty$ and $\phi_{A+}=\phi_{D}$, then $\left\{\pi_{k}\right\}$ is class I if at least one of $\left\{A_{k}\right\}$ and $\left\{D_{k}\right\}$ is class I and class II when both $\left\{A_{k}\right\}$ and $\left\{D_{k}\right\}$ are class II.

(iv) If $1<\eta<\infty$ and $1<\phi_{D}<\eta \leq \phi_{A+}$ or $\eta=\infty$ and $1<\phi_{D}<\phi_{A+}$, then $\left\{\pi_{k}\right\}$ is class I when $\left\{D_{k}\right\}$ is class I and class II when $\left\{D_{k}\right\}$ is class II.

Proof. We will prove part (i); parts (ii), (iii), and (iv) can be proved similarly.

Assume that $1<\eta<\infty$ and $\eta \leq \min \left\{\phi_{A+}, \phi_{D}\right\}$. Since the radius of convergence of

$$
\Pi^{*}(z)=\frac{1}{\operatorname{det}\left(I-R^{*}(z)\right)} \pi_{0} R_{0}^{*}(z) \operatorname{adj}\left(I-R^{*}(z)\right)
$$

is $\eta, \operatorname{det}\left(I-R^{*}(\eta)\right)=0$, and

$$
\pi_{0} R_{0}^{*}(\eta) \operatorname{adj}\left(I-R^{*}(\eta)\right)=\pi_{0} R_{0}^{*}(\eta) v(\eta) u(\eta) \prod_{i=2}^{m}\left[1-r_{i}(\eta)\right] \geq 0
$$

(but not a zero vector, according to Lemma 6 and Lemma 7), $\Pi^{*}(\eta)$ is infinite. Therefore, $\left\{\pi_{k}\right\}$ is class I.

We now discuss the impact of the boundary matrices on the tail asymptotics. It is important to note that the $R$-measure expression (8) shows a subtle interaction between $\left\{A_{k}\right\}$ and $\left\{D_{k}\right\}$ that has a nontrivial influence on the tail behavior of $\left\{\pi_{k}\right\}$. In general, it is possible for $\left\{\pi_{k}\right\}$ to have the tail behavior of any discrete distribution when the boundary matrices determine its tail 
asymptotics, for example under the condition that $1<\phi_{D} \leq \eta<\phi_{A+}$. This phenomenon was also observed by Bean and Nielsen [8] in the tail of the first passage time distribution. Under this condition, explicit tail asymptotics expressions in some special cases can be derived. For example,

(i) if $\phi_{D}<\eta$ and $z=\phi_{D}$ is a pole of order $d$ of $D^{*}(z)$, then, for some small $\varepsilon>0$,

$$
\pi_{k}=\sum_{j=1}^{d}(-1)^{j} \phi_{D}^{-(k+j)}\left(\begin{array}{c}
k+j-1 \\
j-1
\end{array}\right) L_{d-j}+O\left(\left(\phi_{D}+\varepsilon\right)^{-k}\right) e
$$

where

$$
L_{j}=\left.\frac{1}{j !} \frac{\mathrm{d}^{j}}{\mathrm{~d} z^{j}}\left\{\pi_{0} S(z)\left[I-R^{*}(z)\right]^{-1}\right\}\right|_{z=\phi_{D}},
$$

with $S(z)$ analytic in $z,|z|<\phi_{D}+\sigma$ for some $\sigma>0$, such that $S\left(\phi_{D}\right) \neq 0$ and $R_{0}^{*}(z)=S(z) /\left(\phi_{D}-z\right)^{d} ;$ and

(ii) if $\phi_{D}=\eta, z=\phi_{D}$ is a pole of $D^{*}(z)$ of order $d$, and the matrix $A$ is irreducible, then, for some small $\varepsilon>0$,

$$
\pi_{k}=\sum_{j=1}^{d+1}(-1)^{j} \phi_{D}^{-(k+j)}\left(\begin{array}{c}
k+j-1 \\
j-1
\end{array}\right) L_{d+1-j}+O\left(\left(\phi_{D}+\varepsilon\right)^{-k}\right) e .
$$

It is also possible to obtain expressions for the tail asymptotics of $\left\{\pi_{k}\right\}$ when $A^{*}(z)$ and/or $D^{*}(z)$ have algebraic singular points; for example according to the Heaviside operational principle of [29] or to [12].

\section{Concluding remarks}

In this section, we provide an example and some remarks to conclude the paper. In the example, the BMAP/BMAP/1 queue is considered. We show how the tail behavior of the distribution of stationary queue length relates to the tail behavior of the arrival and service processes. In this model, we make the following assumptions.

(i) There is a single server with a waiting room of infinite capacity. Arrivals and service occur in batches. Customers that arrive in different batches are served according to the first-come-first-served discipline, while customers that arrive in the same batch are served with equal probability.

(ii) The arrival process is a BMAP with matrix descriptor $\left\{T_{k}\right\}$, where $T=\sum_{k=0}^{\infty} T_{k}$ is an irreducible infinitesimal generator of size $m \times m, 1 \leq m<\infty$. Let $\pi_{\mathrm{A}}$ be the stationary probability vector of $T$. Then the stationary arrival rate is $\lambda=\pi_{\mathrm{A}} \sum_{k=1}^{\infty} k T_{k} e$.

(iii) The service process is a BMAP with matrix descriptor $\left\{S_{k}\right\}$, where $S=\sum_{k=0}^{\infty} S_{k}$ is an irreducible infinitesimal generator of size $n \times n, 1 \leq n<\infty$. Let $\pi_{\mathrm{S}}$ be the stationary probability vector of $S$. Then the stationary service rate is $\mu=\pi_{\mathrm{S}} \sum_{k=1}^{\infty} k S_{k} e$.

We are interested in the tail asymptotics of the stationary distribution of the queue length. We denote by $(q(t), I(t), J(t))$ the state of the system at time $t$, where, at time $t, q(t), I(t)$, and $J(t)$ are the number of customers in the system, the arrival phase, and the service phase, respectively. The infinitesimal generator of the continuous-time Markov process $\{q(t), I(t), J(t), t \geq 0\}$ is 
GI/G/1 type with repeating and boundary matrix sequences $\left\{A_{k}\right\}$ and $\left\{D_{k}\right\}$ respectively given by

$$
A_{0}=T_{0} \oplus S_{0}, \quad A_{i}=T_{i} \otimes I, \quad i \geq 1, \quad A_{-j}=I \otimes S_{j}, \quad j \geq 1,
$$

and

$$
D_{i}=T_{i} \otimes I, \quad i \geq 0, \quad D_{-j}=I \otimes \sum_{l=j}^{\infty} S_{l}, \quad j \geq 1
$$

Note that

$$
\left(\pi_{\mathrm{A}} \otimes \pi_{\mathrm{S}}\right)\left[\sum_{i=1}^{\infty} i\left(A_{-i}-A_{i}\right)\right] e=\mu-\lambda>0
$$

is a stability condition for the system.

Let

$$
T^{*}(z)=\sum_{k=1}^{\infty} z^{k} T_{k} \quad \text { and } \quad S^{*}(z)=\sum_{k=1}^{\infty} z^{-k} S_{k},
$$

and let $\phi_{T}$ and $\phi_{\mathrm{S}}$ be the radii of convergence of $T^{*}(z)$ and $S^{*}(z)$, respectively. By Theorem 1 and Lemma 3, we have $\phi_{R_{0}}=\phi_{R}=\phi_{T}$. Theorem 2 shows that if $\rho=\lambda / \mu<1$ and $\phi_{T}>1$, then $\left\{q_{k}\right\}$ is light tailed, where

$$
q_{k}=\lim _{t \rightarrow \infty} \mathrm{P}\{q(t)=k\}=\pi_{k} e, \quad k \geq 0
$$

According to Theorems 3, 4, and 5, we obtain the following corollary.

Corollary 2. (i) If $\rho=\lambda / \mu<1, \phi_{T}>1$, and the set

$$
\Omega=\left\{z: 1<|z|<\phi_{T}, \operatorname{det}\left(T_{0} \oplus S_{0}+T^{*}(z) \otimes I+I \otimes S^{*}(z)\right)=0\right\}
$$

is not empty, then the tail asymptotics of $\left\{q_{k}\right\}$ is geometric.

(ii) If $\rho=\lambda / \mu<1, \phi_{T}>1$, and $r\left(\phi_{T}\right)>1$, where $r\left(\phi_{T}\right)$ is the maximal eigenvalue of $\sum_{k=1}^{\infty} \phi_{T}^{k} R_{k}$, then the asymptotics of $\left\{q_{k}\right\}$ is geometric.

Remark 6. If $\left\{T_{k}\right\}$ is light tailed then $\left\{q_{k}\right\}$ is light tailed. In this case, an asymptotic expression of $\left\{q_{k}\right\}$ depends on both $\left\{T_{k}\right\}$ and $\left\{S_{k}\right\}$.

Finally, we remark that expressions for the generating function of $\left\{q_{k}\right\}$ for the matrices of M/G/1 type used in the literature (see, for example, Equation (2.1) of [11] or Equation (3.3.2) of [25]) are not in forms convenient to generalize to the study of the asymptotic behavior of Markov chains of GI/G/1 type.

Following [11], the generating function for Markov chains of GI/G/1 type can be written as

$$
\Pi^{*}(z)\left[I-A^{*}(z)\right]=\pi_{0} D^{*}(z)-\sum_{i=1}^{\infty} z^{i} \pi_{i} \sum_{j=-i}^{\infty} z^{-j} A_{-j} .
$$

For a Markov chain of $\mathrm{M} / \mathrm{G} / 1$ type, this becomes

$$
\sum_{i=1}^{\infty} z^{i} \pi_{i} \sum_{j=-i}^{\infty} z^{-j} A_{-j}=\pi_{1} A_{-1}
$$


and

$$
\Pi^{*}(z)=\left[\pi_{0} D^{*}(z)-\pi_{1} A_{-1}\right]\left[I-A^{*}(z)\right]^{-1},
$$

which has been used in [11] and elsewhere to study the asymptotic behavior of the chain. However, for the Markov chain of GI/G/1 type, it is very difficult to express

$$
\sum_{i=1}^{\infty} z^{i} \pi_{i} \sum_{j=-i}^{\infty} z^{-j} A_{-j}
$$

explicitly. This is why the $R$-measure is effective in explicitly expressing the generating function $\Pi^{*}(z)$ in this paper.

\section{Acknowledgements}

The authors thank the referee for valuable comments and remarks, and acknowledge that this work was partly supported through two research grants from the Natural Sciences and Engineering Research Council of Canada (NSERC) and the National Natural Science Foundation of China (grant no. 90412012).

\section{References}

[1] Abate, J. And Whitt, W. (1997). Asymptotics for M/G/1 low-priority waiting-time tail probabilities. Queueing Systems 25, 173-223.

[2] Aвате, J. And Whitt, W. (1999). Modeling service-time distributions with non-exponential tails: beta mixtures of exponentials. Stoch. Models 15, 517-546.

[3] Abate, J., Choudhury, G. And Whitt, W. (1994). Asymptotics for steady-state tail probabilities in structured Markov queueing models. Stoch. Models 10, 99-143.

[4] Abate, J., Choudhury, G. And Whitt, W. (1994). Waiting-time tail probabilities in queues with long-tailed service-time distributions. Queueing Systems 16, 311-338.

[5] Anderson, W. J. (1991). Continuous-Time Markov Chains. An Applications-Oriented Approach. Springer, New York.

[6] Asmussen, S. (2003). Applied Probability and Queues, 2nd edn. Springer, New York.

[7] Asmussen, S. and Møller, J. R. (1999). Tail asymptotics for M/G/1 type queueing processes with subexponential increments. Queueing Systems 33, 153-176.

[8] Bean, N. G. And Nielsen, B. F. (2002). Decay rates of discrete phase-type distributions with infinitely-many phases. In Matrix-Analytic Methods, eds G. Latouche and P. Taylor, World Scientific, River Edge, NJ, pp. 17-38.

[9] Bean, N. G., Li, J. M. AND TAYLOR, P. G. (2000). Caudal characteristics of QBDs with decomposable phase spaces. In Advances in Algorithmic Methods for Stochastic Models, eds G. Latouche and P. Taylor, Notable Publications, NJ, pp. 37-55.

[10] Choudhury, G. AND Whitt, W. (1994). Heavy-traffic asymptotic expansions for the asymptotic decay rates in the BMAP/G/1 queue. Stoch. Models 10, 453-498.

[11] FAlkenberG, E. (1994). On the asymptotic behavior of the stationary distribution of Markov chains of M/G/1 type. Stoch. Models 10, 75-97.

[12] Flajolet, P. and Odlyzko, A. (1990). Singularity analysis of generating functions. SiAM J. Discrete Math. 3, 216-240.

[13] Fujimoto, K., Takahashi, Y. and Maкimoto, N. (1998). Asymptotic properties of stationary distributions in two-stage tandem queueing systems. J. Operat. Res. Soc. Japan 41, 118-141.

[14] Grassmann, W. K. and Heyman, D. P. (1990). Equilibrium distribution of block-structured Markov chains with repeating rows. J. Appl. Prob. 27, 557-576.

[15] Grassmann, W. K. And Stanford, D. A. (1999). Matrix-analytic methods. In Computing Probability, ed. W. K. Grassmann, Kluwer, Boston, MA, Chapter 6.

[16] Heyman, D. P. (1995). A decomposition theorem for infinite stochastic matrices. J. Appl. Prob. 32, 893-901.

[17] HøjgaArd, B. and Møller, J. R. (1996). Convergence rates in matrix analytic models. Stoch. Models 12, $265-284$.

[18] IshizAKI, F. AND TAKine, T. (2000). Bounds for the tail distribution in a queue with a superposition of general periodic Markov sources: theory and application. Queueing Systems 34, 67-100. 
[19] Latouche, G. and Ramaswami, V. (1999). Introduction to Matrix Analytic Methods in Stochastic Modeling. SIAM, Philadelphia, PA.

[20] LI, Q.-L. AND ZHaO, Y. Q. (2005). Heavy-tailed asymptotics of stationary probability vectors of Markov chains of GI/G/1 type. Adv. Appl. Prob. 37, 482-509.

[21] Markushevich, A. I. (1965). Theory of Functions of a Complex Variable. Prentice-Hall, Englewood Cliffs, NJ.

[22] MøLlER, J. R. (2001). Tail asymptotics for M/G/1 type queueing processes with light-tailed increments. Operat. Res. Lett. 28, 181-185.

[23] Neuts, M. F. (1981). Matrix-Geometric Solutions in Stochastic Models: An Algorithmic Approach. Johns Hopkins University Press, Baltimore, MD.

[24] Neuts, M. F. (1986). The caudal characteristic curve of queues. Adv. Appl. Prob. 18, 221-254.

[25] Neuts, M. F. (1989). Structured Stochastic Matrices of M/G/1 Type and Their Applications. Marcel Dekker, New York.

[26] Neuts, M. F. and TaKahashi, Y. (1981). Asymptotic behavior of the stationary distributions in the GI/PH/c queue with heterogeneous servers. Z. Wahrscheinlichkeitsth. 57, 441-452.

[27] Subramanian, V. AND SRIKANT, R. (2000). Tail probabilities of low-priority waiting times and queue lengths in MAP/GI/1 queues. Queueing Systems 34, 215-236.

[28] Takahashi, Y. (1981). Asymptotic exponentiality of the tail of the waiting-time distribution in a $\mathrm{PH} / \mathrm{PH} / c$ queue. Adv. Appl. Prob. 13, 619-630.

[29] Wilf, H. S. (1994). Generatingfunctionology, 2nd edn. Academic Press, Boston, MA.

[30] ZhaO, Y. Q. (2000). Censoring technique in studying block-structured Markov chains. In Advances in Algorithmic Methods for Stochastic Models, eds G. Latouche and P. Taylor, Notable Publications, NJ, pp. 417-433.

[31] ZhaO, Y. Q., Li, W. And Alfa, A. S. (1999). Duality results for block-structured transition matrices. J. Appl. Prob. 36, 1045-1057.

[32] ZhaO, Y. Q., LI, W. AND Braun, W. J. (1998). Infinite block-structured transition matrices and their properties. Adv. Appl. Prob. 30, 365-384.

[33] Zhao, Y. Q., Li, W. and Braun, W. J. (2003). Censoring, factorizations, and spectral analysis for transition matrices with block-repeating entries. Method. Comput. Appl. Prob. 5, 35-58. 